\title{
AVALIACÃO DE INSTRUMENTOS AGROMETEOROLÓGICOS ALTERNATIVOS PARA O MONITORAMENTO DA AMBIÊNCIA EM GALPÕES AVÍCOLAS
}

\author{
Nerandi Luiz Camerini ${ }^{1}$, Luciano Barreto Mendes ${ }^{2}$, Jeane Karla de Mendonça Mota ${ }^{1}$, José Wallace Barbosa do \\ Nascimento $^{3}$, Dermeval Araújo Furtado ${ }^{3}$
}

\begin{abstract}
RESUMO
Neste trabalho foram realizados dois experimentos em condições de laboratório, sendo o seu objetivo avaliar a eficiência de instrumentos alternativos de baixo custo para a medição de dados agrometeorológicos, quando comparados com os instrumentos padrões, de custo mais elevado. O primeiro experimento descrito consistiu na comparação entre dois tipos de termômetros de bulbo-negro alternativos, com um termômetro de bulbo-negro padrão, confeccionado em cobre. O segundo experimento foi projetado para comparar um psicrômetro alternativo (feito de um psicrômetro de leitura visual, adaptado com a inserção de sensores HOBO de temperatura), com o sensor comercial HOBO de temperatura ambiente e umidade relativa. Para ambos os experimentos, os instrumentos alternativos foram avaliados quanto à precisão, por meio do valor de $\mathrm{R}^{2}$; concomitantemente, a exatidão dos instrumentos alternativos foi avaliada a partir da comparação dos coeficientes angulares (a) e lineares (b) das equações de regressão com os valores considerados padrão. Concluiu-se que todos os instrumentos alternativos apresentaram boa precisão $\left(\mathrm{R}^{2}=0,90-0,94\right.$ para ambos os tipos de globos-termômetros e $\mathrm{R}^{2}=0,8$ para o psicrômetro); observou-se também que, em ambos os experimentos, os instrumentos alternativos apresentaram exatidão satisfatória, podendo ser utilizados em estudos agrometeorológicos, implicando menores custos para a pesquisa.
\end{abstract}

Palavras-chave: avicultura, bioclimatologia, temperatura do globo negro e umidade relativa do ar.

\section{EVALUATION OF ALTERNATIVE AGROMETEOROLOGICAL INSTRUMENTS FOR MONITORING POULTRY SHED ENVIRONMENT}

\begin{abstract}
Laboratory experiments were conducted to evaluate the efficiency of low-cost alternative instruments for measuring agrometeorological data and compare them with the standard instruments of higher cost. In the first experiment two types of alternative black-bulb thermometers were compared with a standard black-bulb copper thermometer. The second experiment was designed to compare a commercial temperature and relative humidity (HOBO) sensor with an alternative psicrometer, made from a psicrometer of visual reading adapted by adding HOBO temperature sensors. The alternative instruments were evaluated for precision, through the $\mathrm{R}^{2}$ value and also for accuracy assessed by comparing angular (a) and linear (b) coefficients of the regression equations with the standard values. All alternative instruments showed good precision $\left(\mathrm{R}^{2}=0.90\right.$ to 0.94 for both types of thermometers and $\mathrm{R}^{2}=0.8$ for psicrometer); and satisfactory accuracy. It was concluded that these instruments can be used in agrometeorological studies, and thus reduce research costs
\end{abstract}

Keywords: poultry, bioclimatology, black globe temperature, air relative humidity.

\section{Recebido para publicação em 16/03/2010. Aprovado em 22/09/2010}

1- Doutorando (a) em Engenharia Agrícola - Construções Rurais e Ambiência, Universidade Federal de Campina Grande, Campina Grande, PB, nerandi@gmail.com, motajkm@yahoo.com.br

2- Eng. Agrícola, Mestrando em Engenharia Agrícola - Construções Rurais e Ambiência, Universidade do Estado de Iowa, Ames, IA, EUA, luckmendes_al2@yahoo.com.br

3- Professor da Unidade Acadêmica de Engenharia Agrícola, Universidade Federal de Campina Grande, Campina Grande - PB, wallace@deag.ufcg.edu.br,dermeval@deag.ufcg.edu 


\section{INTRODUÇÃO}

As pesquisas atuais de monitoramento das condições de conforto térmico na avicultura têm utilizadovários instrumentosmicrometeorológicos alternativos, para a aferição de variáveis como a temperatura de bulbo negro e a umidade relativa do ar.

A temperatura de globo negro, medida por meio deste instrumento, representa, num único valor, os efeitos combinados da energia radiante, temperatura e velocidade do ar, a partir do qual se pode concluir sobre o nível de conforto de um dado ambiente. Um dos índices utilizados para aferir o conforto térmico dos animais é o ITGU, que é calculado com base na temperatura de globo negro e na temperatura de ponto de orvalho. Para a aferição da temperatura de bulbo negro, vários pesquisadores têm usado esferas metálicas pintadas de preto. Tais instrumentos funcionam como receptores para a quantificação da carga radiante incidente sobre animais e plantas, em estudos referentes ao nível de conforto térmico, sob as mais diversas situações ambientais (SOUZA et al., 2002). Esses autores concluíram que, além de apresentar preço mais acessível com relação ao globo negro de cobre, o globo de plástico mostrou correlação satisfatória com o de cobre, com base no Índice de Temperatura de Globo e Umidade e na Carga Térmica de Radiação, índices de conforto térmico, usados como variáveis comparativas.

Bedford \& Warbner (1934), citados por Souza et al. (2002), usaram termômetros de globo negro feitos de material alternativo, em estudos sobre aquecimento e ventilação em habitações.

Por outro lado, a temperatura ambiente e a umidade relativa do ar, que são elementos meteorológicos de grande importância em estudos bioclimatológicos, são aferidas de várias maneiras. Os instrumentos utilizados compreendem desde os mais comuns (termômetros de mercúrio), até aqueles modernos (sensores resistivos) (CUNHA et al.; (2001). Segundo esses autores, tanto os comuns, quanto os de sensores resistivos apresentam desvantagens, o primeiro pela necessidade de atuação de um observador meteorológico, para efetuar as leituras, e o segundo por apresentar custo elevado.
Marin et al. (2000), citado por Cunha et al. (2001), comparando as medidas de um psicrômetro ventilado, confeccionado, com baixo custo, de termopares $\mathrm{Cu}-\mathrm{Co}$ com um sensor comercial Vaisala, conseguiram boa precisão das medidas, com coeficiente de correlação de 0,98 , apresentando dados de umidade relativa do ar mais coerentes, principalmente em condições de extrema umidade.

Sendo assim, a criação e adaptação de equipamentos alternativos para a aferição de tais variáveis meteorológicas contribuem para a redução dos custos da pesquisa, desde que esses instrumentos apresentem qualidade semelhante àqueles considerados como padrão.

Neste trabalho teve-se como objetivos: (1) avaliar a precisão de dois diferentes termômetros de globo negro alternativo $\left(\mathrm{GNA}_{1}\right.$ e $\mathrm{GNA}_{2}$ ) quando comparados com o termômetro de globo negro padrão (GNP); (2) avaliar o psicrômetro alternativo $\left(\mathrm{P}_{\mathrm{A}}\right)$, quando comparado com o sensor comercial de temperatura ambiente e umidade relativa $\mathrm{HOBO}$ $\left(\mathrm{P}_{\text {ново }}\right)$.

\section{MATERIAIS E MÉTODOS}

O experimento foi conduzido nas dependências do Laboratório de Construções Rurais e Ambiência (LaCRA), do Departamento de Engenharia Agrícola, da Universidade Federal de Campina Grande (UFCG), na cidade de Campina Grande-PB.

Foram utilizadas três unidades (repetições) de cada um dos globos-termômetros confeccionados com os seguintes materiais (tratamentos): 1- O globo negro alternativo 1 (GNA $)_{1}$, constituído de uma esfera de plástico resistente (PVC), com diâmetro $0,15 \mathrm{~m}$, revestido com uma camada de spray na cor preto fosco (Figura 1B); 2 - O globo negro alternativo $2\left(\mathrm{GNA}_{2}\right)$, constituído de uma esfera de plástico resistente (PVC), com diâmetro $0,035 \mathrm{~m}$ e também revestido com uma camada de spray na cor preto fosco (Figura 1C). 3 - Para aferir a temperatura do globo padrão (GNP), utilizou-se um termômetro de globo digital (TGD-100, Instrutherm, São Paulo, SP, Brasil) feito de cobre (Figura 1A), com diâmetro de 0,15 $\mathrm{m}$ e espessura de $0,5 \mathrm{~mm}$, no interior do qual foi inserido um termopar tipo $\mathrm{T}$ (cobre-constantan), com extremidade sensível, localizada no seu centro geométrico. $\mathrm{O}$ termopar estava conectado com um 
módulo de medição e controle, modelo CR10X (Campbell Scientific, Inc., Logan, Utah, EUA). Os globos $\mathrm{GNA}_{1}$ e GNA 2 possuíam nos seus centros geométricos um sensor de temperatura $\mathrm{HOBO}$ (Onset Computer Corporation, Pocasset, MA, EUA).

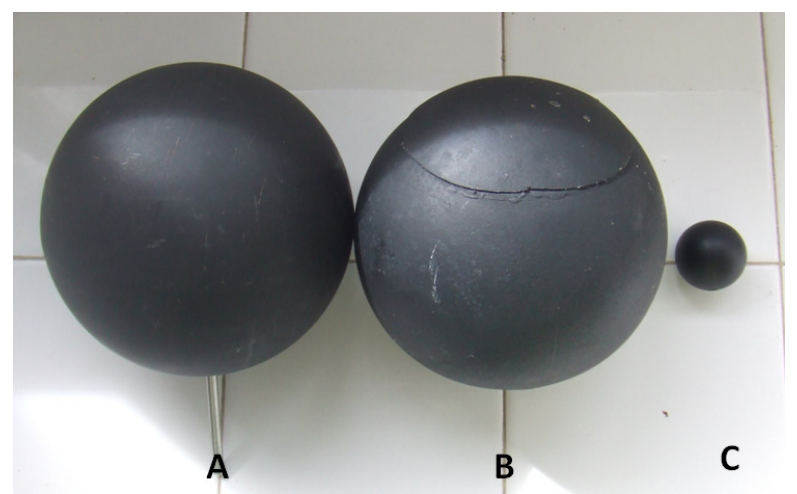

Figura 1. Montagem do sistema de comparação do GNA com o GNP.

Todos os globos foram colocados em um galpão padrão, coberto com telha de fibrocimento $(6 \mathrm{~mm}$ de espessura), para a coleta de dados, juntamente com um termopar tipo T para o monitoramento da temperatura ambiente. Tal sistema lá permaneceu por, aproximadamente, 50 horas. Todos os tipos de globos negros foram programados para armazenar o valor da temperatura do globo a cada 30 minutos.

A análise estatística foi realizada com base nos resultados médios da temperatura de globo para cada tratamento da equação de regressão para definição de fatores de correção dos valores da temperatura de globo, com relação à testemunha (GNP). O grau de ajuste foi medido pelo coeficiente de determinação $\left(\mathrm{R}^{2}\right)$ e por meio dos coeficientes linear (a) e angular (b), os quais traduzem a exatidão máxima quando $\mathrm{a}=0$ e $\mathrm{b}=1$, e a precisão máxima quando $\mathrm{r}^{2}$ tende a 1 .

As medições foram realizadas no Laboratório de Construções Rurais e Ambiência (LaCRA), pertencente ao Departamento de Engenharia Agrícola da Universidade Federal de Campina Grande, na cidade de Campina Grande - PB.

$\mathrm{O}$ experimento consistiu em monitoramento da temperatura ambiente e da umidade relativa do ar, com a utilização de dois tipos diferentes de instrumentos (tratamentos): 1 - psicrômetro alternativo $\left(\mathrm{P}_{\mathrm{A}}\right)$, que consistia em um psicrômetro comercial de leitura visual (Figura 2A) (Incotherm, higrômetro seco-úmido), adaptado com a fixação de sensores de temperatura $\mathrm{HOBO}$, um afixado no lugar do termômetro de bulbo seco e outro afixado no lugar do termômetro de bulbo úmido (Figura 2B). 2 - Sensor de temperatura ambiente e umidade relativa do ar $\mathrm{HOBO}\left(\mathrm{P}_{\text {ново }}\right)$, como ilustrado na Figura 2C.

A coleta de dados ocorreu no interior do laboratório, a uma altura de $1,5 \mathrm{~m}$ do solo.

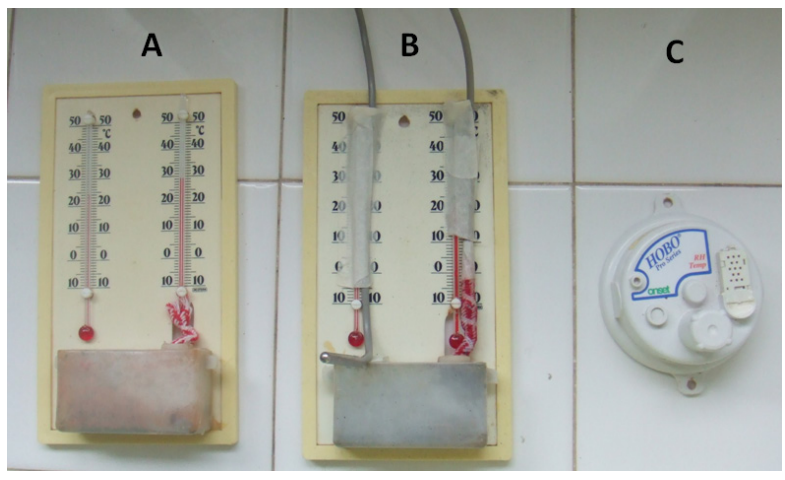

Figura 2. Conjunto psicrométrico de leitura visual utilizado como suporte (A), montagem do psicrômetro alternativo - $\mathrm{P}_{\mathrm{A}}(\mathrm{B})$ e detalhe do sensor de temperatura ambiente $\mathrm{e}$ umidade relativa $\mathrm{HOBO}$ - $\mathrm{P}_{\text {ново }}(\mathrm{C})$.

A umidade relativa pode ser calculada pela Equação 1:

$$
\begin{aligned}
& U R=\frac{e_{a}}{e_{s}} 100 \% U R=\frac{e_{a}}{\theta_{s}} 100 \% \\
& e_{a}=e_{s u}-A P\left(t_{s}-t_{u}\right) \\
& e_{a}=e_{s u}-A P\left(t_{s}-t_{u}\right) \\
& e_{s u}=0,611 \cdot 10^{\left(\frac{7, s, t_{u}}{237,3+t_{u}}\right)} \\
& e_{s}=0,611 \cdot 10^{\left(\frac{7, s t_{s}}{237,3+t_{s}}\right)}
\end{aligned}
$$

em que.

UR - umidade relativa do ar em \%; $\mathrm{e}_{\mathrm{a}}$ - pressão parcial de vapor d'água, em $\mathrm{kPa}$; $\mathrm{e}_{\mathrm{s}}$ - pressão de saturação de vapor d'água à temperatura de bulbo seco, em KPa;

$\mathrm{e}_{\mathrm{su}}$ - pressão de saturação de vapor d'água à temperatura de bulbo úmido, em $\mathrm{kPa}$;

A - constante psicrométrica para junção com ventilação natural $\left(80.10^{-5} \mathrm{C}^{-1}\right)$; 
P - pressão atmosférica local $(95,30 \mathrm{kPa})$;

$\mathrm{t}_{\mathrm{s}}$ - temperatura do sensor de bulbo seco, em ${ }^{\circ} \mathrm{C}$;

$\mathrm{t}_{\mathrm{u}}$ - temperatura do sensor de bulbo úmido, em ${ }^{\circ} \mathrm{C}$.

A coleta dos dados médios da temperatura ambiente e da umidade relativa $\left(\mathrm{P}_{\text {ново }}\right)$ e da temperatura de bulbo seco e bulbo úmido $\left(\mathrm{P}_{\mathrm{A}}\right)$ foi realizado a cada 60 minutos. O tempo total de coleta de dados foi de 97 horas.

A verificação do funcionamento do conjunto psicrométrico $\left(\mathrm{P}_{\mathrm{A}}\right)$ foi feita por meio da correlação com os valores de umidade relativa, obtidos pelo sensor HOBO $\left(\mathrm{P}_{\text {НОВо }}\right)$, utilizando-se o processo de regressão linear simples, sendo que o grau de ajuste foi medido pelo coeficiente de determinação $\left(\mathrm{R}^{2}\right)$ e por meio dos coeficientes linear (a) e angular (b), os quais traduzem a exatidão máxima quando $a=0$ e b

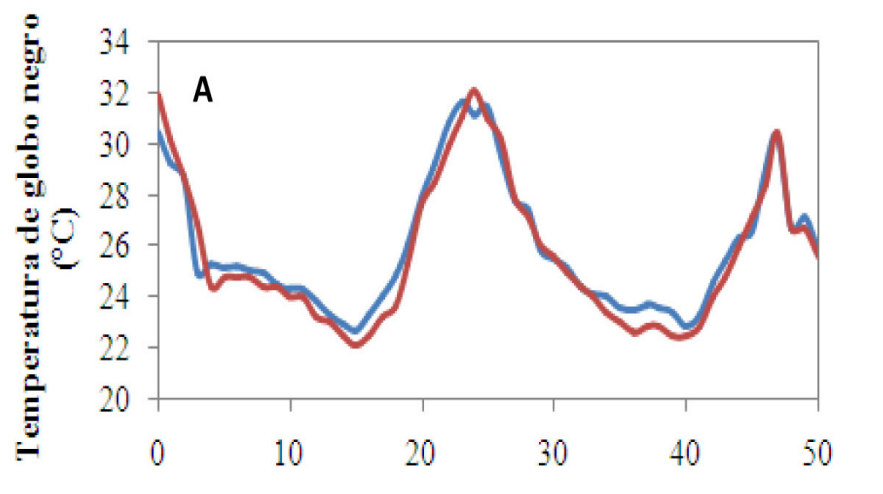

Tempo (horas)
$=1$, e a precisão máxima quando $\mathrm{R}^{2}$ tende a 1 .

\section{RESULTADOS E DISCUSSÕES}

Na Figura 3, observa-se o comportamento dos dados experimentais ao longo do tempo, os picos de máximo e mínimo representam as oscilações naturais de temperatura entre os dias e as noites. Nota-se, ainda, que a $\mathrm{T}_{\mathrm{GNP}}$ esteve predominantemente acima da $\mathrm{T}_{\text {GNA1 }}$ e predominantemente abaixo da $\mathrm{T}_{\text {GNA2 }}$ respectivamente; e os valores médios da temperatura do ar foram 24,6, 25,4 e 24,9 ${ }^{\circ} \mathrm{C}$ para o GNA1, GNA2 e GNP, respectivamente. Nota-se, portanto, que tais médias para o valor da temperatura de globo negro situam-se bem próximas para os três sistemas comparados.

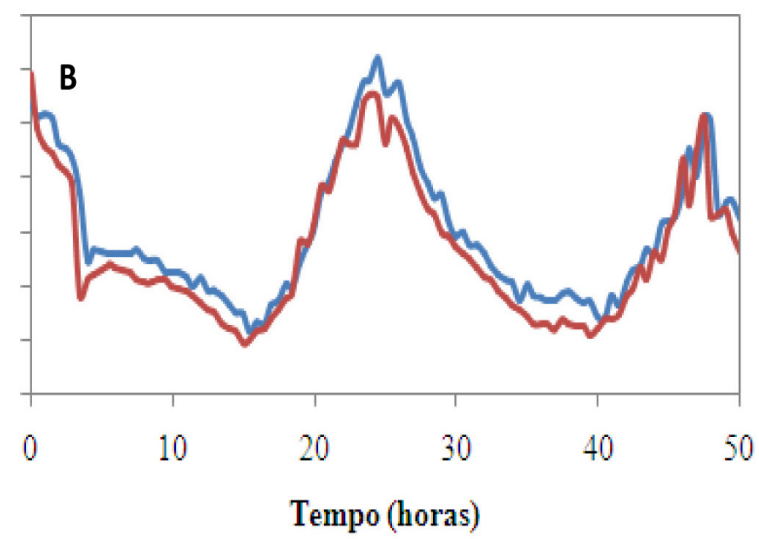

$-\mathrm{GNP}-\mathrm{GNA} 1-\mathrm{GNA} 2$

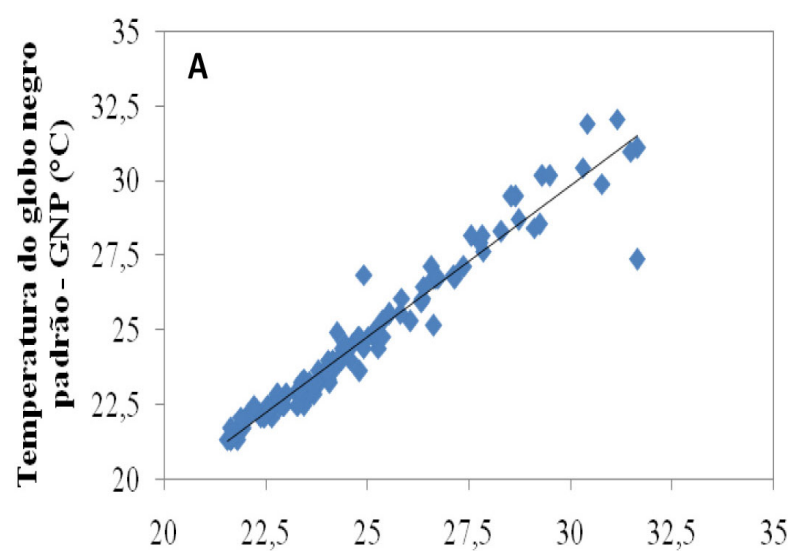

Temperatura do globo negro alternativo 1 - GNA1 $\left({ }^{\circ} \mathrm{C}\right)$

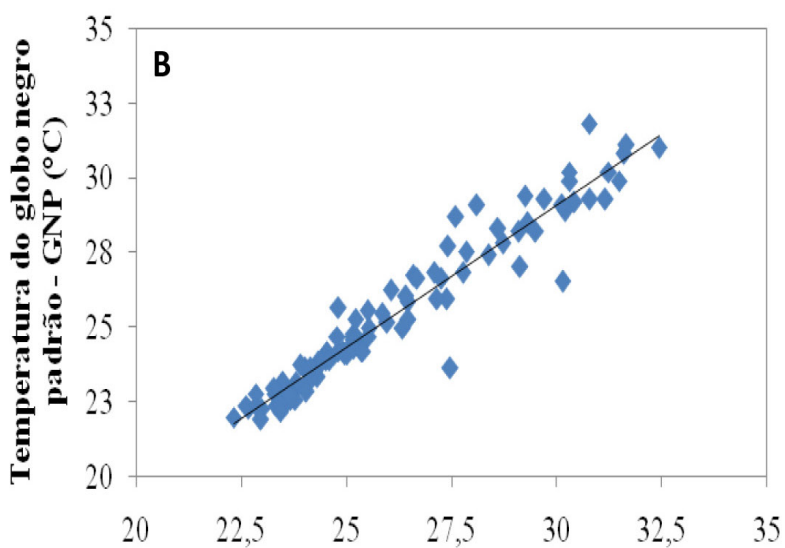

Temperatura do globo negro alternativo 2 - GNA2 $\left({ }^{\circ} \mathrm{C}\right)$

Figura 3. Representação gráfica do comportamento dos dados ao longo do tempo (acima). Representação gráfica dos dados experimentais, obtidos dos termômetros-globos alternativos 1 e $2-\mathrm{T}_{\mathrm{GNA} 1} \mathrm{e}$ $\mathrm{T}_{\mathrm{GNA} 2}$ (A e B, respectivamente), com relação ao termômetro-globo padrão - $\mathrm{T}_{\mathrm{GNP}}$ (abaixo).

\section{REVENG


Ainda na Figura 3, podem-se observar as retas construídas com base nas equações apresentadas no Quadro 2; nesses gráficos, verifica-se a distribuição dos dados experimentais em torno do modelo linear obtido no processo de regressão. As equações de regressão para a temperatura de globo negro padrão, expressas em termos das temperaturas de globo negro, medidas pelos apresentados termômetrosglobos alternativos, encontram-se no Quadro 1.

Os resultados expressos no Quadro 1 concordam com o que foi apresentado por Souza et al. (2002), que, ao compararem termômetrosglobos feitos de diferentes materiais com o termômetro-globo padrão de cobre, também obtiveram os melhores resultados para o modelo equivalente ao do tratamento GNA.

Com relação à correspondência entre $\mathrm{o}$ termômetro-globo padrão e o termômetroglobo alternativo 2 ( $\mathrm{GNA}_{2}$ ), nota-se, no Quadro 1, que, apesar da determinação ter sido menor que a equação da $\mathrm{GNA}_{1}, \mathrm{R}^{2}=0,90$ é ainda considerado como satisfatório, evidenciando que os dois modelos alternativos possuem boa precisão, quando comparados com o modelo padrão. Analisando a exatidão dos instrumentos alternativos quando comparados com o padrão, nota-se que os coeficientes angulares das equações no Quadro 1 estão muito próximos do valor ideal, ou seja, 1; os coeficientes lineares, por sua vez, são valores situados na casa dos décimos e, portanto, muito próximos do valor ideal, ou seja, 0. Tais observações conferem boa exatidão aos instrumentos alternativos avaliados.

Nos gráficos da Figura 4, observa-se o comportamento temporal da umidade do ar e da temperatura ambiente, aferidos pela média dos psicrômetros alternativos $\left(\mathrm{P}_{\mathrm{A}}\right)$ e pelo sensor HOBO $\left(\mathrm{P}_{\text {ново }}\right)$. Para a umidade relativa do ar, nota-se que os valores aferidos pelo $\mathrm{P}_{\text {ново }}$ ficaram sempre abaixo dos valores aferidos pelo $\mathrm{P}_{\mathrm{A}}$; já para a temperatura ambiente os valores aferidos pelo $\mathrm{P}_{\mathrm{A}}$ situaram-se, predominantemente, acima daqueles aferidos pelo $\mathrm{P}_{\text {ново }}$ Os valores médios de umidade relativa do ar foram de 79,0 e 85,2\%, para o $\mathrm{P}_{\text {ново }}$ e o $\mathrm{P}_{\mathrm{A}}$, respectivamente; e os valores médios da temperatura do ar foram de 23,8 e $23,7{ }^{\circ} \mathrm{C}$, para o $\mathrm{P}_{\text {ново }}$ е о $\mathrm{P}_{\mathrm{A}}$, respectivamente. Nota-se, portanto, que embora os valores para a temperatura ambiente tenham sido bem próximos para os dois sistemas comparados, essa diferença é maior quando se trata da umidade relativa do ar. Julga-se, todavia, que essa discrepância tenha sido resultante da atribuição de um valor fixo para a pressão atmosférica local (vide Equação 2), utilizado para o cálculo da umidade relativa, quando, na realidade, esse valor oscilava em torno do valor considerado. Sendo assim, essa maior discrepância de valores para a umidade relativa não pode, a rigor, ser considerada como razão para o descarte do psicrômetro alternativo avaliado.

Quadro 1. Equações de regressão para a correspondência entre as temperaturas $T_{\mathrm{GNA} 1}$ e $\mathrm{T}_{\mathrm{GNA2}}$ e as temperaturas $\mathrm{T}_{\mathrm{GNP}}$. As equações são seguidas de seus coeficientes de determinação

\begin{tabular}{ccc}
\hline Tratamento & Equação & $\mathbf{R}^{2}$ \\
\hline $\mathrm{GNA}_{1}$ & $\mathrm{~T}_{\mathrm{GNP}}=1,010 \mathrm{~T}_{\mathrm{GNA}}-0,489$ & 0,94 \\
$\mathrm{GNA}_{2}$ & $\mathrm{~T}_{\mathrm{GNP}}=0,955 \mathrm{~T}_{\mathrm{GNA} 2}-0,500$ & 0,90 \\
\hline
\end{tabular}

Quadro 2. Equações de regressão para a correspondência entre as umidades relativas $U_{\mathrm{HOBO}_{\mathrm{O}}}$ e $U_{\mathrm{A}}$ e entre as temperaturas $\mathrm{T}_{\text {ново }}$ e $\mathrm{T}_{\mathrm{A}}$. As equações são seguidas de seus coeficientes de determinação

\begin{tabular}{ccc}
\hline Tratamento & Equação & $\mathbf{R}^{\mathbf{2}}$ \\
\hline $\mathrm{UR}$ & $\mathrm{UR}_{\text {ново }}=1,208 \mathrm{UR}_{\mathrm{A}}-23,90$ & 0,80 \\
$\mathrm{~T}$ & $\mathrm{~T}_{\text {ново }}=0,808 \mathrm{~T}_{\mathrm{A}}+4,567$ & 0,81 \\
\hline
\end{tabular}



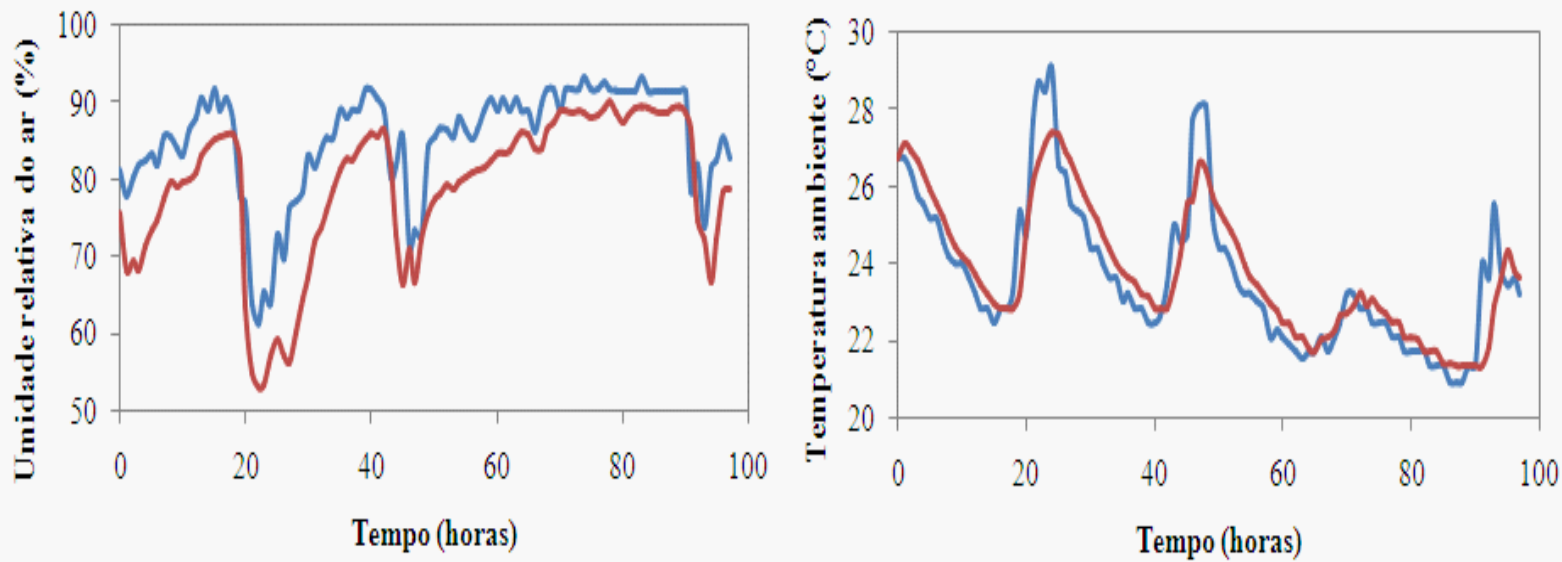

$-\mathrm{PA}-\mathrm{PHOBO}$


Figura 4. Representação gráfica do comportamento dos dados de umidade relativa e temperatura ambiente para os psicrômetros $\mathrm{P}_{\mathrm{A}}$ e $\mathrm{P}_{\text {ново }}$ ao longo do tempo (acima). Representação gráfica dos dados experimentais, obtidos do psicrômetro PA (A : UR e B : $T_{\text {ambiente }}$, respectivamente), com relação ao sensor padrão $\mathrm{P}_{\text {ново }}$ (abaixo).

Ainda, na Figura 4, vê-se a correlação entre os valores medidos pelo instrumento alternativo e pelo instrumento padrão, utilizando-se os dados do período analisado. A simples observação dos gráficos da figura dá ideia da dispersão dos dados em torno do modelo linear de regressão encontrado. Para melhor entender essa dispersão e correlação, as Equações de regressão e os coeficientes de determinação podem ser vistos no Quadro 2. Tais coeficientes podem ser considerados satisfatórios para ambas as variáveis, a umidade relativa do ar e a temperatura do ar ambiente.

Sendo assim, com a observação dos dados do Quadro 2, nota-se que houve boa concordância entre o psicrômetro alternativo analisado, e o sensor HOBO, para ambas as variáveis aferidas, o que confere precisão satisfatória ao psicrômetro alternativo. Deve-se notar, porém, que houve tendência à superestimativa da umidade relativa na ordem de $8,5 \%$ do valor de UR fornecido pelo sensor HOBO, ao passo que houve tendência à subestimativa do valor da temperatura ambiente na ordem de 2,6\% do valor de $\mathrm{T}$, fornecido pelo sensor HOBO. Com relação à precisão do psicrômetro alternativo, nota-se que essa pode ser considerada como satisfatória, pela observação dos coeficientes angulares das equações no Quadro 2. Já o coeficiente linear da equação que representa a

\section{REVENG


correlação entre os valores de temperatura é bem mais próximo do valor ideal $(0)$, do que o coeficiente linear da equação para a umidade relativa.

\section{CONCLUSÕES}

- Com relação aos termômetros-globos avaliados, nas condições em que o trabalho foi conduzido, concluiu-se que ambos os modelos alternativos $\left(\mathrm{GNA}_{1}\right.$ e $\left.\mathrm{GNA}_{2}\right)$ representam opções satisfatórias para substituição do termômetro de globo padrão (GNP); sendo assim, pela precisão e exatidão avaliadas, constituem alternativa viável cujo custo é acessível; e

- $\mathrm{O}$ conjunto psicrométrico alternativo $\left(\mathrm{P}_{\mathrm{A}}\right)$, avaliado nas condições do experimento mencionado, apresenta boa concordância dos valores medidos de temperatura e umidade relativa do ar, em relação ao sensor comercial HOBO, possibilitando a sua utilização em estudos agrometeorológicos, com baixo custo.

\section{REFERÊNCIAS BIBLIOGRÁFICAS}

BEDFORD, T.; WARNER, C. The globe thermometer in studies of heating and ventilation. Journal of Hygiene, v.34, n.3, p.458-473, Mar. 1934.

CAMPBELL SCIENTIFIC, INC. CR10X Datalogger: Operator's manual. Logan, Utah: Copyright (C), 1984-2000.

CUNHA, A.R.; ESCOBEDO, J.F.; GALVANI, E. Avaliação de um psicrômetro de termopar de baixo custo. Revista Brasileira de Agrometeorologia, Santa Maria, RS, v.9, n.1, p.17-22, 2001.

MARIN, F.R.; ANGELOCCI, L.R.; VILA NOVA, N.A. Construção de psicrômetro de termopar de baixo custo e verificação de seu desempenho em condições de campo. In: REUNIÓN ARGENTINA DE AGROMETEOROLOGÍA, 8., LA AGROMETEOROLOGÍA HACIA EL SIGLO 21. CD-ROM, 2000, Mendoza. Mendoza, Argentina: Associación Argentina de Agrometeorologia, 2000.

SOUZA, C.F.; TINÔCO, I.F.F.; BAÊTA, F.C; FERREIRA, W.P.M.; SILVA, R.S. Avaliação de materiais alternativos para confecção do termômetro de globo. Cienc. Agrotec., Lavras, v.26, n.1, p.157-164, jan./fev., 2002. 